\title{
Robust Unit Commitment Considering Uncertain Demand Response
}

\author{
G. Liu ${ }^{\mathrm{a}, *}$, K. Tomsovic ${ }^{\mathrm{b}}$ \\ ${ }^{a}$ Power and Energy Systems Group, Oak Ridge National Laboratory, One Bethel Valley \\ Road, P.O. Box 2008, MS-6070, Oak Ridge, TN 37831-6070, USA \\ ${ }^{b}$ Department of Electrical Engineering and Computer Science, The University of \\ Tennessee, Knoxville, 1520 Middle Drive, Knoxville, TN 37996, USA
}

\begin{abstract}
Although price responsive demand response has been widely accepted as playing an important role in the reliable and economic operation of power system, the real response from demand side can be highly uncertain due to limited understanding of consumers' response to pricing signals. To model the behavior of consumers, the price elasticity of demand has been explored and utilized in both research and real practice. However, the price elasticity of demand is not precisely known and may vary greatly with operating conditions and types of customers. To accommodate the uncertainty of demand response, alternative unit commitment methods robust to the uncertainty of the demand response require investigation. In this paper, a robust unit commitment model to minimize the generalized social cost is proposed for the optimal unit commitment decision taking into account uncertainty of the price elasticity of demand. By optimizing the worst case under proper robust level, the unit commitment solution of the proposed model is robust against all possible realizations of the modeled uncertain demand response. Numerical simulations on the IEEE Reliability Test System show the effectiveness of the method. Compared to unit commitment with deterministic price elasticity of demand, the proposed robust model can reduce the average Locational Marginal Prices (LMPs) as well as the price volatility.
\end{abstract}

Keywords: Unit commitment, price elasticity, demand response, uncertainty, robust optimization, Locational Marginal Price (LMP)

\footnotetext{
*Corresponding author. Tel.: +1 865773 5812; fax.: +1 8655749329.

Email addresses: liug@ornl.gov (G. Liu), tomsovic@utk.edu (K. Tomsovic)
} 


\section{Nomenclature}

The main symbols used in this paper are defined below. Others will be defined as required in the text.

\subsection{Indices and Numbers}

$i \quad$ index of generators, running from 1 to $N_{G}$.

$j \quad$ index of demand, running from 1 to $N_{D}$.

$t \quad$ index of time periods, running from 1 to $N_{T}$.

$k \quad$ index of transmission lines, running from 1 to $N_{K}$.

$m \quad$ index of energy blocks offered by generators (demand), running from 1 to $N_{I}\left(N_{J}\right)$.

\subsection{Variables}

0.2.1. Binary Variables

$u_{i t} \quad 1$ if unit $i$ is scheduled on during period $t$ and 0 otherwise.

$u_{j t} \quad 1$ if demand $j$ is scheduled to be reduced during period $t$ and 0 otherwise.

\subsubsection{Continuous Variables}

$p_{i t}(m) \quad$ power output scheduled from the $m$-th block of energy offer by unit $i$ during period $t$. Limited to $p_{i t}^{\max }(m)$.

$d_{j t}(m) \quad$ demand reduction from the $m$-th block of demand $j$ 's reduction curve during period $t$. Limited to $d_{j t}^{\max }(m)$.

$P_{i t} \quad$ power output scheduled from unit $i$ during period $t$.

$D_{j t} \quad$ demand reduction for demand $j$ during period $t$.

$\lambda_{j t} \quad$ corresponding price when demand $j$ during period $t$ is reduced by $D_{j t}$.

$R_{i t} \quad$ scheduled spinning reserve for unit $i$ during time period $t$.

$\tilde{\alpha}_{j t} \quad$ a random variable of the slope of price elastic demand reduction curve of demand $j$ during period $t$. 


\subsection{Constants}

$\lambda_{i t}(m) \quad$ marginal cost of the $m$-th block of energy offer by unit $i$ during period $t$.

$m c_{j t}(m)$ marginal opportunity or alternative cost of the $m$-th block of demand $j$ 's reduction curve during period $t$.

$A_{i} \quad$ operating Cost of unit $i$ at the point of $P_{i}^{\min }$.

$B_{j} \quad$ opportunity or alternative cost demand $j$ when it is reduced by $D_{j}^{\min }$.

$C_{i t} \quad$ capacity cost offer of unit $i$ during period $t$ for providing upspinning reserve.

$P_{i}^{\max } \quad$ maximum output of unit $i$.

$P_{i}^{\min } \quad$ minimum output of unit $i$.

$D_{j t}^{\max } \quad$ maximum reduction of demand $j$ during period $t$.

$D_{j t}^{\min } \quad$ minimum reduction of demand $j$ during period $t$.

$\lambda_{j t}^{\max } \quad$ corresponding price when demand $j$ during period $t$ is reduced by $D_{j t}^{\max }$.

$\lambda_{j t}^{\min } \quad$ corresponding price when demand $j$ during period $t$ is reduced by $D_{j t}^{\min }$.

$\lambda_{j t}^{\text {ref }} \quad$ reference price when demand $j$ during period $t$ is not reduced .

$\alpha_{j t} \quad$ the slope of price elastic demand reduction curve of demand $j$ during period $t$.

$e_{j t}^{m} \quad$ the $m$-th elbow point of the piece-wise linear price elastic demand reduction curve of demand $j$ during period $t$

$\triangle \alpha_{j t} \quad$ deviation from the nominal slop of price elastic demand reduction curve of demand $j$ during period $t$.

$D_{j t}^{F} \quad$ fixed demand of demand $j$ during period $t$.

$D_{j t}^{\text {ref }} \quad$ reference responsive demand of demand $j$ during period $t$ without reduction.

$G S F_{k i} \quad$ generation shift factor to line $k$ from unit $i$.

$G S F_{k j} \quad$ generation shift factor to line $k$ from demand $j$.

$F_{k}^{\max } \quad$ transmission limit of line $k$.

$\Gamma_{0} \quad$ control parameter of robustness level. 


\section{Introduction}

Following the deregulation of power system, electricity prices in the wholesale market have at times experienced dramatic and rapid changes. This price volatility may arise from market power or an imbalance in supply and demand stemming from, for example, loss of generation units, unit ramping constraints, transmission lines outages or congestion and sudden load changes. When renewable energy resources, such as, wind and solar, are introduced, this problem can become worse, e.g., frequent negative electricity prices occur in the western region of ERCOT [1]. Under these circumstances, demand response (DR) programs, which can change the energy consumption patterns of consumers, could improve market efficiency and reduce price volatilities. DR is implemented for obtaining reliable and efficient electricity markets in several countries [2]-[4].

Considerable efforts have been devoted to incorporating DR into the market clearing process to achieve the highest efficiency. In [5], an electricity market in which generators and consumers can submit offers and bids on both energy and reserve are proposed, but the network and multi-period constraints are neglected. In [6] and [7], a price elasticity matrix (PEM) is proposed and taken into consideration when scheduling generation and setting the pool price. An iterative market clearing algorithm is used and the demand is adjusted in proportion to the difference between market clearing price and the reference price. In [8], a day-ahead market clearing tool is proposed for the load shifting behavior of consumers by submitting price sensitive bids. The effect of DR on the market are quantified and analyzed. In [9], price responsive demand shift bidding of consumers is introduced in a day-ahead market with network constraints. A linear price-elastic demand curve is used to represent the sensitivity of demand with respect to price. DR with inter-temporal characteristics is incorporated into a security constrained unit commitment (SCUC) for economic and security purposes in [10]. The price-elastic demand curve is approximated as a stepwise linear curve. DR participation in the spinning reserve market is also investigated in [11]-[13].

In order to eliminate the barrier of DR participating in electricity market, Federal Energy Regulatory commission (FERC) issued Order No. 719 in 2008 [14]. By this order, FERC requires that ISOs/RTOs accept bids from qualified demand response resources to provide ancillary services. In addition, aggregators on behalf of small retail customers are allowed to bid DR directly into the organized markets. Currently, several ISOs/RTOs (e.g. California ISO, ERCOT, ISO-New England, Midwest ISO, PJM, and New York 
ISO) have provided opportunities for customers to participate in wholesale energy, capacity and ancillary services markets [15]. Taking New York ISO for example, DR resources may offer operating reserves, regulation, energy reduction and capacity service by participating into the Demand Side Ancillary Services Program, Day-Ahead Demand Response Program, Emergency Demand Response program and Installed Capacity Special Case Resources Program [16]. By 2010, 31,695 MW of demand response are available in ISO/RTO markets, up from 17,146 MW at the end of 2006. Such gains represent $6.6 \%$ of 2008 peak demand within the regions combined [14].

In the above literature and market practice, DR directly bids into various markets and is modeled as a deterministic price-elastic demand curve. However, the actual price-elastic demand curve is uncertain and variable in time. In addition, consumers may modify their demand as prices change without being centrally dispatched. Therefore, power system scheduling, particularly unit commitment (UC), needs to be robust against the uncertainty in the price elasticity of demand. In recent years, significant contribution has been made by using the stochastic optimization models to solve UC problem under various uncertainties, in particular, under wind power output uncertainty [17]-[20]. A stochastic UC model is developed to determine the optimal reserve levels considering the volatile wind power in [21]. The impacts of largescale wind power on system operating cost, realisability and environment are fully assessed in [22]. In [23], Benders decomposition technique is used to solve the stochastic UC problem. A chance-constrained two-stage stochastic UC with uncertain wind power output is proposed in [24]. Nevertheless, stochastic UC is rarely used in real system operation for two reasons. Firstly, the realization of uncertainty by a large number of scenarios dramatically increase the dimension of optimization model and reduce the solution efficiency. Secondly, the exact wind distribution is rarely available in short-term, such as day-ahead. For these reasons, robust optimization model, which requires less information of the uncertain parameter and has high solution efficiency, has been proposed to solve the UC problem with uncertainty recently. Two-stage robust UC models have been developed to solve the day-ahead UC problem under load uncertainty in [25] and [26], wind power output uncertainty in [27], generator and transmission uncertainty in [28] and market price uncertainty in [29]. For robust UC, the uncertainties are expressed by deterministic uncertainty sets neglecting their probability distributions and the worst case is optimized. Compared to stochastic UC, robust UC has relatively low dimension and high solution efficiency. 
Considering the uncertainty of demand response, a scenario set of demand price elasticities is proposed in [30] to represent the stochastic DR, i.e., customers have different responses to the electricity prices in different scenarios, but the probability distribution of the demand elasticities is difficult to quantify. In [31] and [32], the price elasticity of demand is assumed to be varying within a given range. A robust $\mathrm{UC}$ approach is proposed to maximize the social welfare under the worst case joint wind power output and price-elastic demand curve scenario. This method allows for increased demand elasticity and results in a paradoxical reduction of total social welfare. This is because social welfare is not the right index of evaluating the economic benefit of system with demand response. The calculated social welfare is reduced as a consequence of increased demand elasticity [33]. Under this situation, the worst scenario in term of maximizing social welfare in [31] and [32] appears to be the one with the most price-elastic demand. This is clearly incorrect since greater demand elasticity leads to more reliable and/or efficient operation of the system due to the flexibility of demand.

In view of the above concerns, the main contribution of this paper is to propose a new robust UC model to correct for the inconsistency that occurs when the effect of uncertain demand elasticity on social welfare is calculated. In the proposed model, the objective is to minimize the generalized social cost, which consists of generation cost and opportunity cost of reduced demand or alternative cost of electricity consumption. In this model, a low elasticity of demand results in increased generalized social costs and more generation capacity is needed, which is consistent with practical system operation. A robust UC model is developed to take into account the uncertain price elasticity of demand. The proposed robust UC model can be used for the reliability unit commitment run process (e.g., reliability assessment commitment in Midwest ISO) to ensure sufficient resources are available and online to cover uncertainties. Numerical simulations show that the proposed model can reduce both the average electricity price and the volatility of prices.

The rest of this paper is organized as follows. In Section II, the proposed UC model to minimize generalized social cost is formulated. The robust UC model considering uncertainty demand elasticity is developed in Section III. Results of numerical simulations are presented in Section IV. Finally, conclusions are given in Section V. 


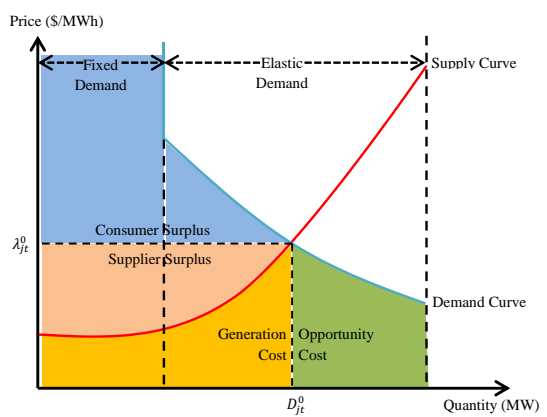

(a) Deterministic demand curve

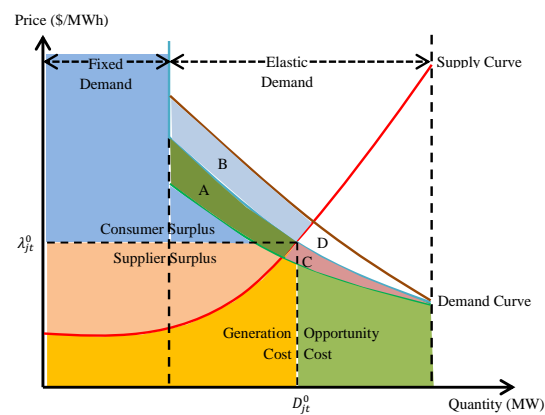

(b) Uncertain demand curve

Figure 1: Market equilibrium with deterministic and uncertain demand curves

\section{Proposed UC Model to Minimize Generalized Social Cost}

In this section, we first compare the proposed UC for minimizing generalized social cost with a traditional UC for maximizing social welfare. The mathematical formulation of proposed UC is presented. Consumers have the opportunity to reduce their electricity charges by adjusting their activities according to the market clearing prices; however, some consumers will not have the ability or sufficient incentive to adjust demand at a given price. Accordingly, demand is divided into fixed and price elastic components.

A typical market equilibrium with deterministic demand curve is shown in Fig. 1a. The maximum social welfare, which consists of consumers' surplus and suppliers' surplus, occurs at the intersection of the electricity supply and demand curves $\left(D_{j t}^{0}, \lambda_{j t}^{0}\right)$. At the same point, the generalized social cost, which consists of generation cost and opportunity cost of the reduced demand or the alternative cost of electricity consumption is minimized. The generalized social cost can be seen as the complement of social welfare. When the social welfare is maximized, the generalized social cost is minimized and vice versa. In other words, with a deterministic supply and demand curves, a UC that minimizes generalized social cost is equivalent to a UC that maximizes social welfare. The mathematical proof of the equivalence between maximizing social welfare and minimizing generalized social cost under deterministic demand curves is given in Appendix A.

When the demand curve is uncertain, as depicted in Fig. 1b, minimizing generalized social cost may no longer be equivalent to maximizing social welfare. The worst scenario in term of maximizing social welfare is when the demand curve is green with a flat slope, i.e., the price elasticity is highest, so that the social welfare is reduced by area $\mathrm{A}$ and the needed generation 
capacity is the minimum of all scenarios. In contrast, the worst scenario in term of minimizing generalized social cost is when the demand curve is grey with the greatest slope, i.e., the price elasticity is lowest, so that the generalized social cost is the increased by area $\mathrm{D}$ and the needed generation capacity is the maximum of all scenarios. In fact, the worst scenario in term of minimizing generalized social cost is the best scenario in terms of maximizing social welfare and vice versa. The mathematical proof can be found in Appendix B.

The proposed UC model for minimizing generalized social cost is the correct approach when the demand curve is uncertain. In practice, with demand becoming more price-elastic, the reliability and/or efficiency of system can be improved due to the extra flexibility from demand side. In other words, the worst case in actual system operation should be when the demand is the least elastic, i.e., the price elasticity of demand is the lowest. This is in contradiction with the traditional UC model for maximizing social welfare, where the worst scenario is the highest price elasticity. In this traditional model, the scenario with the most price-elastic demand is optimized and the committed generation capacity is the minimum of all scenarios. When the real price elasticity of demand is low, the generation capacity will be insufficient and electricity price spikes may appear. This is the opposite of what is desired from a robust optimization. As to the proposed UC model for minimizing generalized social cost, the worst scenario is the one with the lowest price elasticity. In the proposed model, the scenario with the least price-elastic demand is optimized and the committed generation capacity is the maximum of all scenarios. This is consistent with practical system operation. Therefore, the proposed UC model is more appropriate than the traditional UC model when the demand curve is uncertain.

It should be emphasized that the proposed UC model differs from the traditional UC model only when the demand curve is uncertain. With uncertainty, there is an area, including A, B, C and D, where the demand curve can be realized. Consequently, the intersection of the electricity supply and demand curves changes from a single point into a curve. Under this situation, the complementarity between social welfare and generalized social cost no longer exists. In other words, maximizing social welfare is no longer equivalent to minimizing generalized social cost with uncertain demand response.

The proposed UC model to minimize the generalized social cost with 
deterministic demand curve (price elasticity) is as follows:

$$
\begin{gathered}
\min \quad \sum_{t=1}^{N_{T}} \sum_{i=1}^{N_{G}}\left[\sum_{m=1}^{N_{I}} \lambda_{i t}(m) p_{i t}(m)+A_{i} u_{i t}\right] \\
+\sum_{t=1}^{N_{T}} \sum_{j=1}^{N_{D}}\left[\sum_{m=1}^{N_{J}} m c_{j t}(m) d_{j t}(m)+B_{j} u_{j t}\right] \\
+\sum_{t=1}^{N_{T}} \sum_{i=1}^{N_{G}} S_{i t}\left(u_{i t}, u_{i, t-1}\right) \\
+\sum_{t=1}^{N_{T}} \sum_{i=1}^{N_{G}} C_{i t} R_{i t} \\
P_{i t}=\sum_{m=1}^{N_{I}} p_{i t}(m)+u_{i t} P_{i}^{\min } \quad \forall i, \forall t \\
0 \leq p_{i t}(m) \leq p_{i t}^{\max }(m) \quad \forall i, \forall t \forall m \\
D_{j t}=\sum_{m=1}^{N_{J}} d_{j t}(m)+u_{j t} D_{j t}^{\min } \quad \forall j, \forall t \\
0 \leq d_{j t}\left(m_{i} \leq d_{j t}^{\max }(m) \quad \forall j, \forall t, \forall m\right. \\
\sum_{i=1}^{N_{G}} P_{i t}=\sum_{j=1}^{N_{D}}\left(D_{j t}^{F}+D_{j t}^{\mathrm{ref}}-D_{j t}\right) \quad \forall t \\
\sum_{j=1}^{N_{G}} G S F_{k j}\left(D_{j t}^{F}+D_{j t}^{\mathrm{ref}}-D_{j t}\right) \mid \leq F_{k}^{\max } \quad \forall k, \forall t \\
R_{i t}+P_{i t} \leq P_{i}^{\max } u_{i t} \quad \forall i, \forall t \\
P_{i t}+R_{i t} \leq \sum_{i=1}^{N_{G}} R_{i t} \quad \forall i, \forall t
\end{gathered}
$$$$
\text { s.t. }
$$

In the above formulation, the objection function (1) is the generalized social cost, including energy cost of generators (line 1), opportunity cost of reduced demand (line 2), startup cost of generators (line 3) and spinning reserve cost (line 4). All terms are in mixed-integer linear form except the startup cost of generators (line 3 ), which can be simply recast into mixedinteger linear form as in [34]. Constraints (2) and (3) approximate the energy cost of generators by blocks [35]. Similarly, the opportunity cost of reduced demand is linearized and approximated by (4) and (5). The market equilibrium is enforced by (6). A DC power flow is used to represent the transmission constraints in (7). The coupling relationship between energy and spinning reserve is represented by constraint (8). The minimum amount of spinning 
reserve based on " $n-1$ " contingency rule is ensured by (9) [36]. For more comprehensive approach to quantifying spinning reserve, see [37]. Additionally, each unit or demand is subject to its own operating constraints, including minimum up and down time, initial condition, capacity limits and ramp limits. See [38] for more details about the formulations of these constraints.

\section{Robust UC with Uncertain Price Elasticity of Demand}

In this section, we first introduce a price elastic demand reduction curve to represent the price elasticity. Then, the model of uncertain price elasticity is introduced. The robust UC with uncertain price elasticity of demand is formulated.

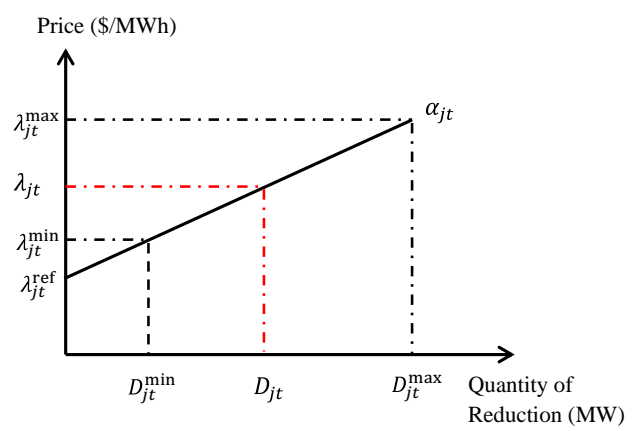

Figure 2: A typical price elastic demand reduction curve

A typical price elastic demand reduction curve is shown in Fig. 2. The demand reduction increases monotonically with electricity price increase. The slope of the curve $\alpha_{j t}$ represents the price elasticity of demand. The electricity price $\lambda_{j t}$ of a consumer corresponding to reduction of demand by $D_{j t}$ can be represented as:

$$
\lambda_{j t}=\lambda_{j t}^{\mathrm{ref}}+\alpha_{j t} D_{j t}
$$

The opportunity cost or alternative cost of reduced demand can be determined by the integral of the curve from 0 to $D_{j t}$.

$$
C_{j t}\left(D_{j t}\right)=\lambda_{j t}^{\mathrm{ref}} D_{j t}+0.5 \alpha_{j t}\left(D_{j t}\right)^{2}
$$

The quadratic cost function (11) can be approximated by piecewise linear functions similar to that for generation units [34]. The marginal cost $m c_{j t}(m)$ 
can be represented as

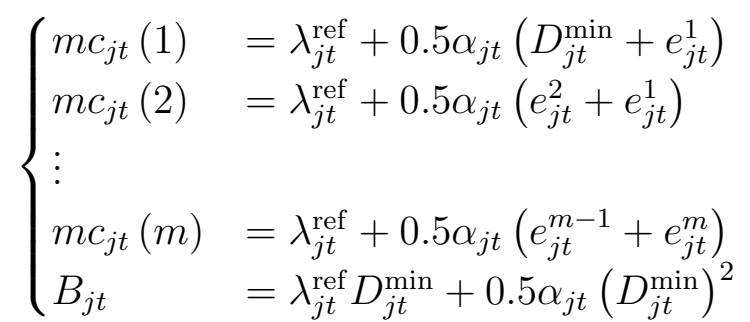

In the proposed UC with deterministic price elasticity, we have deterministic estimates for the slope $\alpha_{j t}$ of the price elastic demand reduction curve. The parameter $\alpha_{j t}$ is very hard to quantify or estimate as mentioned before. Here, each $\alpha_{j t}$ is modeled as an independent, symmetric and bounded random variable (but with unknown distribution) $\tilde{\alpha}_{j t}$, that falls within $\left[\alpha_{j t}-\triangle \alpha_{j t}, \alpha_{j t}+\triangle \alpha_{j t}\right]$, where $\triangle \alpha_{j t} \geq 0$.

To formulate a robust UC, we introduce an integer control parameter $\Gamma_{0}$, which takes values within the interval $\left[0,\left|J_{0}\right|\right]$, where $J_{0}=\left\{(j t) \mid \triangle \alpha_{j t}>0\right\}$, i.e., $\alpha_{j t}$ is subject to parameter uncertainty $\left(\alpha_{j t}=\tilde{\alpha}_{j t}\right)$ for all $(j t) \in J_{0}$. The parameter $\Gamma_{0}$ controls the level of robustness in the objective. We are interested in finding a solution that optimizes against all scenarios under which a number $\Gamma_{0}$ of price elasticities can vary in such a way as to maximally increase the objective function. If $\Gamma_{0}=0$, the uncertainty of price elasticity is completely ignored, while if $\Gamma_{0}=\left|J_{0}\right|$, uncertainties in price elasticity are fully considered, leading to the most conservative solution [29][39].

For a demand with uncertain price elasticity, the opportunity cost of reduced demand in the worst scenario is (13), where $e_{j t}^{0}=D_{j t}^{\min }$ and $e_{j t}^{N J}=$ $D_{j t}^{\max }$. Considering all demands with uncertain price elasticity, we can obtain the robust counterpart of equation (1) as (14).

$$
\begin{aligned}
& \sum_{m=1}^{N J}\left[\lambda_{j t}^{\mathrm{ref}}+0.5\left(\alpha_{j t}+\triangle \alpha_{j t}\right)\left(e_{j t}^{m-1}+e_{j t}^{m}\right)\right] d_{j t}(m) \\
& +\lambda_{j t}^{\mathrm{ref}} D_{j t}^{\mathrm{min}}+0.5\left(\alpha_{j t}+\triangle \alpha_{j t}\right)\left(D_{j t}^{\mathrm{min}}\right)^{2} u_{j t} \\
= & \sum_{m=1}^{N J} m c_{j t}(m) d_{j t}(m)+B_{j} u_{j t} \\
& +\triangle \alpha_{j t}\left|u_{j t}\left(e_{j t}^{0}\right)^{2} / 2+\sum_{m=1}^{N J} d_{j t}(m)\left(e^{m-1}+e^{m}\right) / 2\right|
\end{aligned}
$$




$$
\begin{aligned}
\min & \sum_{t=1}^{N_{T}} \sum_{i=1}^{N_{G}}\left[\sum_{m=1}^{N_{I}} \lambda_{i t}(m) p_{i t}(m)+A_{i} u_{i t}\right] \\
& +\sum_{t=1}^{N_{T}} \sum_{j=1}^{N_{D}}\left[\sum_{m=1}^{N_{J}} m c_{j t}(m) d_{j t}(m)+B_{j} u_{j t}\right] \\
& +\sum_{t=1}^{N_{T}} \sum_{i=1}^{N_{G}}\left[S_{i t}\left(u_{i t}, u_{i, t-1}\right)+C_{i t} R_{i t}\right] \\
& +\max _{\left\{S_{0}\left|S_{0} \subseteq J_{0},\right| S_{0} \mid \leq \Gamma_{0}\right\}} \sum_{(j t) \in S_{0}}\left\{\triangle \alpha_{j t} \mid u_{j t}\left(e_{j t}^{0}\right)^{2} / 2\right. \\
& \left.+\sum_{m=1}^{N_{J}} d_{j t}(m)\left(e^{m-1}+e^{m}\right) / 2 \mid\right\}
\end{aligned}
$$

By the property of strong duality, the proposed robust UC with uncertain price elasticity of demand can be reformulated as (15)-(21). A detailed description of how to convert the nonlinear objective function (14) into a mixed integer one as (15) can be found in Appendix C [39].

It should be noted that the proposed robust UC only considers selfelasticities of demand. To take deterministic cross-elasticities into account, the deterministic UC model (1)-(9) becomes a mixed integer quadratic programming (MIQP) [40]. To extend the proposed robust UC model to consider the uncertain cross-elasticities into account, the quadratic terms in objective function can be transformed into quadratic constraints. Correspondingly, the MILP model (15)-(21) will become a mixed integer quadratic constrained programming (MIQCP). For simplicity, only self-elasticities are considered in this paper.

$$
\begin{aligned}
\min & \sum_{t=1}^{N_{T}} \sum_{i=1}^{N_{G}}\left[\sum_{m=1}^{N_{I}} \lambda_{i t}(m) p_{i t}(m)+A_{i} u_{i t}\right] \\
& +\sum_{t=1}^{N_{T}} \sum_{j=1}^{N_{D}}\left[\sum_{m=1}^{N_{J}} m c_{j t}(m) d_{j t}(m)+B_{j} u_{j t}\right] \\
& +\sum_{t=1}^{N_{T}} \sum_{i=1}^{N_{G}}\left[S_{i t}\left(u_{i t}, u_{i, t-1}\right)+C_{i t} R_{i t}\right]+\sum_{t=1}^{N_{T}} \sum_{j=1}^{N_{D}} q_{j t}+z_{0} \Gamma_{0}
\end{aligned}
$$

s.t.

(2) to $(9)$

$$
\begin{gathered}
z_{0}+q_{j t} \geq \triangle \alpha_{j t} y_{j t} \quad \forall j t \in J_{0} \\
q_{j t} \geq 0 \quad \forall j t \in J_{0}
\end{gathered}
$$




$$
\begin{gathered}
y_{j t} \geq 0 \quad \forall j t \in J_{0} \\
-y_{j t} \leq x_{j t} \leq y_{j t} \quad \forall j t \in J_{0} \\
x_{j t}=u_{j t}\left(e_{j t}^{0}\right)^{2} / 2+\sum_{m=1}^{N_{J}} d_{j t}(m)\left(e^{m-1}+e^{m}\right) / 2 \quad \forall j t \in 0
\end{gathered}
$$

The solution methodology used in this paper is from [39], which is also utilized in [31] and [32]. The min-max problem is converted into a monolithic mixed integer linear programming problem by dualizing the inner maximization problem. Unlike [31] and [32], only uncertainty of demand price elasticity is considered in our model, which results in uncertainty only in the objective function. Thus, dualization of the inner maximization problem does not generate nonlinear terms, and the whole optimization model can be solved by MILP. In [31] and [32], uncertainties from both demand response and wind are taken into account, which appears in both objective function and constraints. Dualization of the inner max-min problem generates bilinear terms. Thus, Benders' Decomposition algorithm is used to solve the multi-stage optimization problem. In other word, the solution methodology in this paper can be seen as a simplified version of that in [31] and [32].

\section{Case Studies}

The proposed robust UC model is demonstrated on a modified IEEE Reliability Test System [41]. In the modified system, there are 17 demand nodes, 26 thermal generators and the hydro units have been removed. The ramp rates and quadratic cost coefficients are taken from [42]. The unit quadratic cost curves in [42] are converted into piece-wise linear cost curves. In addition, we assume that all units offer spinning reserve at the rate of $10 \%$ of their highest incremental cost for producing energy [43].

The analysis is conducted for a 24-hour scheduling horizon and each time interval is set to one hour. The forecasted demand is taken from Table 2 in [42]. The UC with forecasted demand is solved and the LMPs are calculated by minimizing the generation cost. These LMPs are set as the reference prices

$\lambda_{j t}^{\text {ref }}$ of the price elastic demand reduction curves as in Fig. 2. Then, each demand is divided into two parts: fixed and price elastic with a proportion of $80 \%$ and $20 \%$, respectively. The price elasticity is set to $0.2 \$ / \mathrm{MW}^{2} \mathrm{~h}[9]$. Based on these parameters, the price elastic demand reduction curves of the form in Fig. 2 are determined. The minimum reduced demand is set to be $50 \%$ of the price elastic portion. The opportunity cost of reduced demand is 
calculated and then linearized into piece-wise linear segments.

All numerical simulations are coded in MATLAB and solved using the MILP solver CPLEX 12.2. With a pre-specified duality gap of $0.1 \%$, the running time of each case is about 20 seconds on a $2.66 \mathrm{GHz}$ Windows-based PC with $4 \mathrm{G}$ bytes of RAM.

\subsection{Effect of Robustness Level}

In order to show the effect of $\Gamma_{0}$, we assume the slope of the demand reduction curves for all load falls within $[0.1,0.3] \$ / \mathrm{MW}^{2} \mathrm{~h}$. In addition, the robustness level is defined as $\Gamma_{0} /\left(N_{T} \times N_{J}\right)$, where 0 means no robustness and 1 means fully robust. For different robust levels, the generalized social costs are calculated and shown as in Fig. 3.

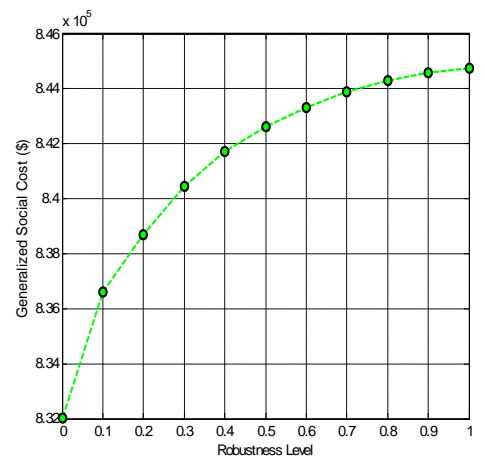

Figure 3: Effect of Robustness Level

As can be seen in Fig. 3, the generalized social cost monotonically increases, but not linearly with the robustness level. We are trying to find an optimal solution that optimizes against all scenarios under which a number $\Gamma_{0}$ of price elasticities can vary in such a way as to maximally increase the objective function. Specifically, when the robust level is low, i.e., $\Gamma_{0}$ is small, the larger demands, which have major impact on the objective function, are considered. As a result, the generalized social cost and the solution robustness increase quickly. When the robust level is high, i.e., $\Gamma_{0}$ is big, the smaller demands, which have a minor impact on the objective function, are also taken into account. In this case, the generalized social cost and the robustness of solution increase more slowly. Thus, there is a diminishing return for considering greater uncertainty.

A set of 50 randomly sampled slopes of the demand reduction curves are generated based on a uniform distribution in the interval $[0.1,0.3] \$ / \mathrm{MW}^{2} \mathrm{~h}$. For the sampling of elasticities, we assume all load have the same demand 
elasticity to make it easier to see the impact, but we could easily consider correlated probabilistic models of demand price elasticity at different nodes. For each UC solution, an economic dispatch problem is formulated for generators committed and demands scheduled for reduction. The social welfare is maximized with constraints, including energy balance, transmission limits (DC power flow), spinning reserve requirements and other capacity limits. The economic dispatch problem is solved by linear programming and the LMPs are obtained as a byproduct. After solving the economic dispatch problem for all samples, the average LMPs over all nodes are calculated and reported. To mimic the high cost of dispatching fast-start units or involuntary load shedding in the real-time operation, a slack variable is added to the total spinning reserve constraint and the corresponding penalty factor is set to be $500 \$ / \mathrm{MW}$. The average total social welfare is calculated and shown in Table 1. It should be noted that the benefit of fixed demand is neglected because it is a large positive constant, so the average social welfare values here are negative.

As can be seen, the proposed robust solution has higher average social welfare than deterministic solution. The highest social welfare happens when robust level is 0.1 (increased by $4.06 \%$ ). As observed, the robust solution performs best when the robust level is relatively small. This can be explained by the central limit theorem. When a large number of independent random variables, $N_{T} \times N_{J}$, are aggregated, the volatility scales according to $\sqrt{N_{T} \times N_{J}}$. Therefore, a proper level of robust level should be chosen as $\frac{O\left(\sqrt{N_{T} \times N_{J}}\right)}{N_{T} \times N_{J}}[26]$. In this paper, with $N_{T}=24$ and $N_{J}=17,2 \times \frac{\sqrt{N_{T} \times N_{J}}}{N_{T} \times N_{J}} \approx 0.1$, which is close to the best robust level. Thus, the proposed robust solution with the proper robustness level can improve the economic performance of the system. For the rest of case studies, we set the robust level as 0.4 .

\subsection{Robustness against Uncertainty of Price Elasticity}

The unit commitment results of the proposed robust and deterministic UC are compared in Fig. 4. As can be seen, more units are committed at hours 7, 8 and 19-22 by the proposed robust method because the approach considers the worst-case scenario of demand price elasticities. In this worstcase scenario, the price elasticity of demand is over-estimated and the real price elasticity of DR is much lower as in Fig. 1b. As a result, the generation capacity is insufficient and electricity price spikes appear. By optimizing for this scenario, more units must be committed and additional generation capacity made available. Consequently, the electricity spikes are reduced. 
Table 1: Average total social welfare for different robust levels

\begin{tabular}{|c|c|c|c|c|c|}
\hline $\begin{array}{c}\text { Robust } \\
\text { Level }\end{array}$ & $\begin{array}{c}\text { Average } \\
\text { Social } \\
\text { Welfare }(\$)\end{array}$ & $\begin{array}{c}\text { Robust } \\
\text { Level }\end{array}$ & $\begin{array}{c}\text { Average } \\
\text { Social } \\
\text { Welfare }(\$)\end{array}$ & $\begin{array}{c}\text { Robust } \\
\text { Level }\end{array}$ & $\begin{array}{c}\text { Average } \\
\text { Social } \\
\text { Welfare }(\$)\end{array}$ \\
\hline 0 & -440791.28 & 0.35 & -423070.14 & 0.70 & -423472.23 \\
\hline 0.05 & -422897.75 & 0.40 & -423211.38 & 0.75 & -423472.23 \\
\hline 0.10 & -422889.20 & 0.45 & -423211.38 & 0.80 & -423472.23 \\
\hline 0.15 & -422929.87 & 0.50 & -423472.23 & 0.85 & -423472.23 \\
\hline 0.20 & -423076.85 & 0.55 & -423472.23 & 0.90 & -423472.23 \\
\hline 0.25 & -423074.24 & 0.60 & -423472.23 & 0.95 & -423472.23 \\
\hline 0.30 & -423074.24 & 0.65 & -423472.23 & 1.00 & -423472.23 \\
\hline
\end{tabular}

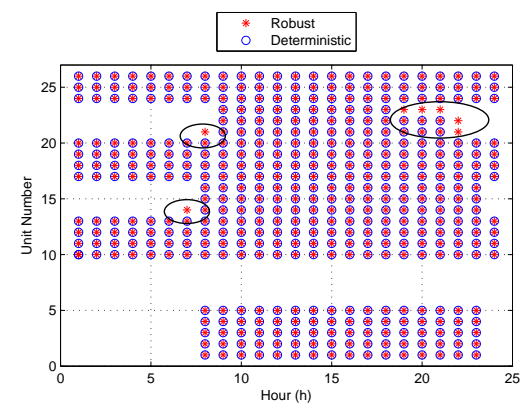

Figure 4: Comparison of unit status between proposed robust and deterministic methods

A set of 50 randomly sampled slopes of the demand reduction curves are generated based on a uniform distribution in the interval $[0.1,0.3] \$ / \mathrm{MW}^{2} \mathrm{~h}$. From the unit commitment results of these two methods, the LMPs are calculated for each sample slope and shown in Fig. 5. In Fig. 5a, the vertical bars show the range of LMPs and the mark on each bar shows the average value. Compared to the deterministic UC, the proposed robust UC shows reduced variation of LMPs. Comparison of mean and standard deviation of LMPs between the proposed robust and deterministic methods is shown in Fig. 5b. Generally, both the average and standard deviation of LMPs are reduced by the proposed method.

The unit commitment results of the proposed robust UC with different penetration of responsive demand are compared in Fig. 6. As can be seen, when the penetration level of responsive demand is increased to $40 \%$, less units are committed during hour 7.The reason is, with higher penetration level of responsive demand, more flexibility will be provided from the de- 


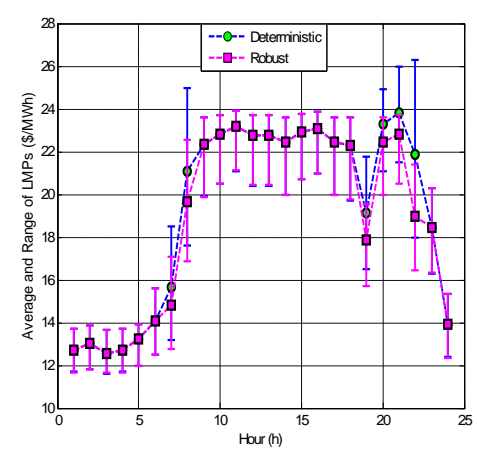

(a) Average and range
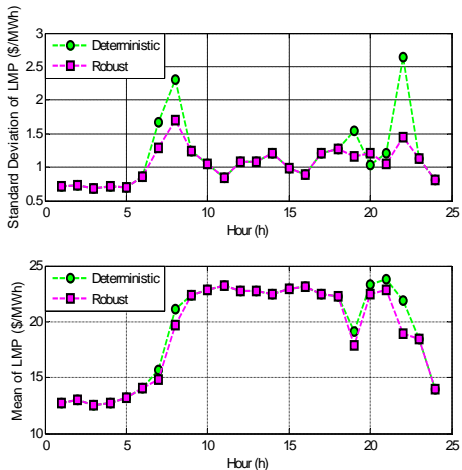

(b) Mean and standard deviation

Figure 5: Comparison of LMPs between proposed robust and deterministic methods

mand side. Therefore, the committed capacity from generators is reduced. Nevertheless, given the same penetration level of responsive demand, the proposed robust UC always commits more generation capacity compared to deterministic UC as the price of increased robustness.

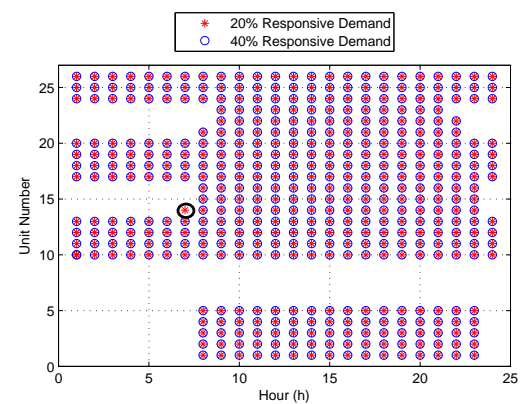

Figure 6: Comparison of unit status with different penetration of responsive demand

\subsection{Comparison of Robust UC to Maximize Social Welfare with Determin- istic $U C$}

The unit commitment results of robust $\mathrm{UC}$ with objective to maximize social welfare and deterministic UC are compared in Fig. 7. Compared to the deterministic UC, fewer units are committed during hours 9-21 and 2324 by the robust UC to maximize social welfare. This is because the worst scenario in terms of maximizing social welfare is when the price elasticity of demand is the highest as in Fig. 1b. By optimizing for this scenario, fewer 
units are committed. Still, if the real price elasticity of demand is lower than the estimated one, the generation capacity will be insufficient and electricity price spikes may appear. This can be seen from Fig. 8a and 8b.

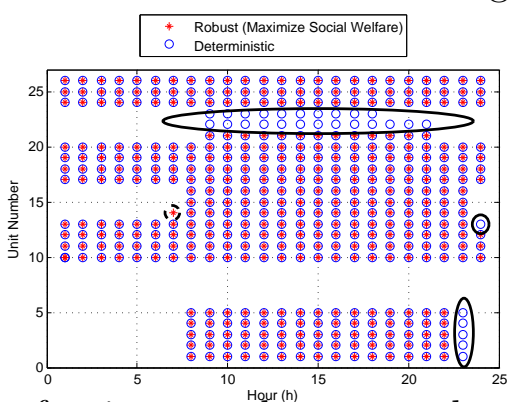

Figure 7: Comparison of unit status between robust UC to maximize social welfare and deterministic UC

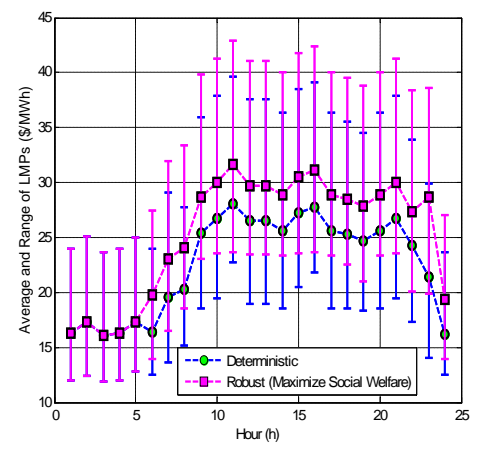

(a) Average and range
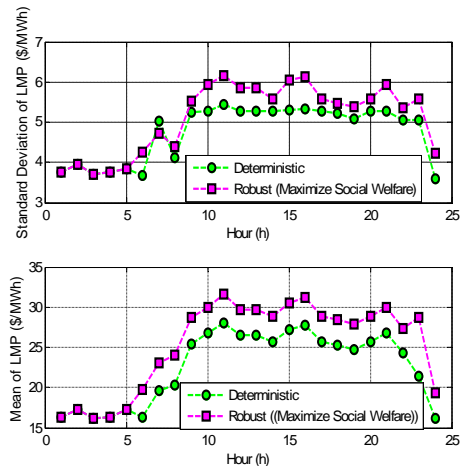

(b) Mean and standard deviation

Figure 8: Comparison of LMPs between robust UC to maximize social welfare and deterministic UC

A set of 50 randomly sampled slopes of the demand reduction curves are generated based on a uniform distribution in the interval $[0.1,0.3] \$ / \mathrm{MW}^{2} \mathrm{~h}$. From the unit commitment results of these two methods, the LMPs are calculated for each sample slope and shown in Fig. 8a. Compared to the deterministic UC, the robust UC with objective to maximize social welfare shows increased variation of LMPs. Comparison of mean and standard deviation of LMPs is shown in Fig. 8b. As can be seen, both the average and standard deviation of LMPs are higher by the robust UC with objective to maximize social welfare.

To summarize, the worst scenario for the robust UC to maximize social welfare is different from the worst scenario for the proposed robust UC to 
minimize generalized social cost. For robust UC to maximize social welfare, the worst scenario is when the price elasticity is the highest. By optimizing this scenario, fewer units are committed, and electricity price spikes will be exacerbated due to insufficient generation capacity. For the proposed robust UC to minimize generalized social welfare, the worst scenario is when the price elasticity is the lowest. By optimizing this scenario, more units are committed, and electricity price spikes will be reduced due to sufficient generation capacity. Thus, the proposed robust UC to minimize generalized social cost can obtain more robust UC decisions.

\subsection{Robustness against Different Distributions of Price Elasticity}

As mentioned before, the probability distribution for demand price elasticity is difficult to estimate. Thus, it is important for a UC solution to be robust against various distributions. Instead of uniform distribution, uncertain price elasticities with normal distribution are tested in this subsection, a new set of 50 randomly sampled slopes for the demand reduction curves is generated that follows a normal distribution with mean $0.2 \$ / \mathrm{MW}^{2} \mathrm{~h}$ and standard deviation $0.2 / 2.88 \$ / \mathrm{MW}^{2} \mathrm{~h}$. This results in $85 \%$ of samples falling between $[0.1,0.3]$ and negative samples are ignored [26]. Based on the unit commitment results of these two methods, the LMPs are calculated for each sample of price elasticities and shown in Fig. 9a. Compared to the deterministic UC, the proposed robust UC significantly reduces volatility.

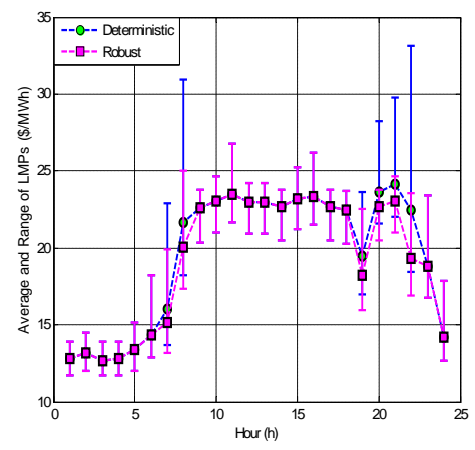

(a) Average and range
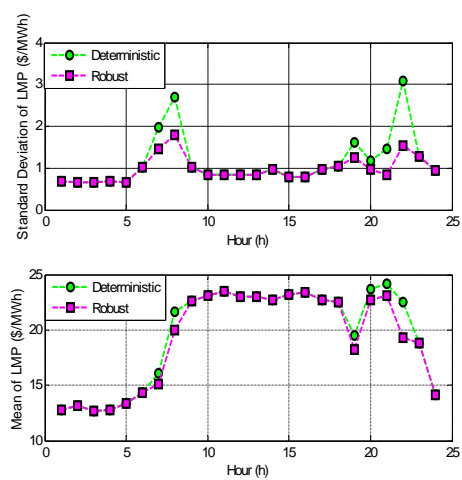

(b) Mean and standard deviation

Figure 9: Comparison of LMPs under normally distributed price elasticities

The mean and standard deviation of LMPs are calculated and shown in Fig. 9b. As can be seen, both the average and standard deviation of LMPs by the proposed robust method are reduced. The proposed UC method is 
robust against different probability distributions in elasticities.

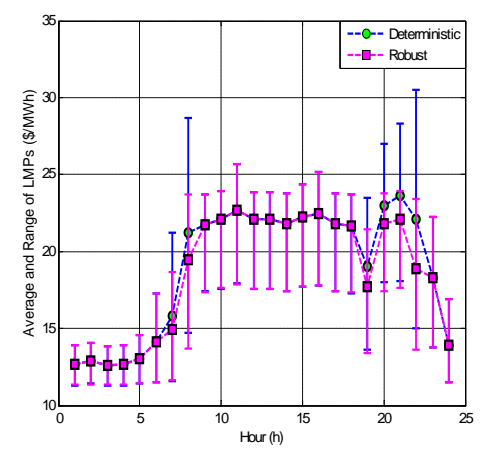

(a) Average and range
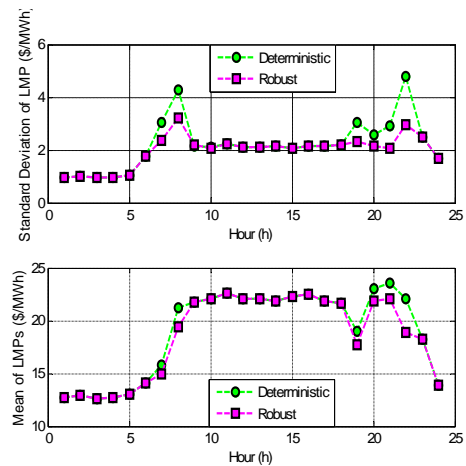

(b) Mean and standard deviation

Figure 10: Comparison of LMPs under uniformly distributed price elasticities with high forecast error

\subsection{Robustness Against High Forecast Errors of Price Elasticity}

In practice, the interval of demand price elasticity may also be difficult to accurately estimate. When the forecast errors of price elasticity are much higher than the estimation, i.e., the real interval of elasticity is much larger than the one used in the UC, it is important for the solution to remain robust. To evaluate this, the UC is solved for elasticity in a uniformly distributed interval of $[0.1,0.3] \$ / \mathrm{MW}^{2} \mathrm{~h}$ and tested against the interval $[0.01,0.39] \$ / \mathrm{MW}^{2} \mathrm{~h}$. The LMPs are calculated for each sample and shown in Fig. 10a. Compared to the deterministic UC, the proposed robust UC significantly reduces the price volatility even though the estimated interval is imprecise.

The mean and standard deviation of LMPs are calculated and shown in Fig. 10b. As can be seen, both the average and standard deviation of LMPs are reduced compared to that of the deterministic method. Thus, the proposed UC method is robust against when the range of price elasticity is poorly understood.

\section{Conclusions}

In this paper, a robust UC model to minimize the generalized social cost is proposed. Compared to the traditional UC to maximize the social welfare, the proposed UC model more effectively manages uncertainty in the demand response. The UC solution of the proposed model is robust against all possible modeled realizations of the uncertain demand response. 
Numerical simulation results on the IEEE Reliability Test System show that the solution can reduce both average prices and price volatility relative to the deterministic UC. In addition, the proposed UC method is robust against different probability distributions and high forecast errors in the demand elasticity.

Although we only consider the uncertainty of demand price elasticity in this paper, the proposed robust UC model can be easily expanded to include other uncertainties, such as, power output of renewable energy resources. First, the power output of renewable energy resources can be assumed within a given interval, which can be determined based on historical data and current weather condition. The price elasticity of demand curve is also varying in a range. Then, the objective is to minimize the generalized social cost under the joint worst-cast scenario which takes account of all uncertainties. Multistage robust optimization model can be used to realize the uncertainties in different stages. Dualization of the inner optimization problem may generate bilinear terms. In that case, Benders' Decomposition algorithm can be used to solve the multi-stage robust optimization problem. Space limits prevent us from exploring these uncertainties further in this paper.

\section{Appendix A. Proof of the equivalence between two UC models under deterministic demand curves}

Based on the proposed UC model to minimize the generalized social cost with deterministic demand curve (1) - (10), we are going to prove this model is equivalent to the traditional UC that maximizes social welfare. The function of generalized social cost is:

$$
\begin{aligned}
F_{S C}\left(x_{S C}\right)= & \sum_{t=1}^{N T} \sum_{i=1}^{N G}\left[\sum_{m=1}^{N I} \lambda_{i t}(m) p_{i t}(m)+A_{i} u_{i t}\right] \\
& +\sum_{t=1}^{N T} \sum_{j=1}^{N D}\left[\sum_{m=1}^{N J} m c_{j t}(m) d_{j t}(m)+B_{j} u_{j t}\right] \\
& +\sum_{t=1}^{N T} \sum_{i=1}^{N G} S_{i t}\left(u_{i t}, u_{i, t-1}\right)+\sum_{t=1}^{N T} \sum_{i=1}^{N G} C_{i t} R_{i t}
\end{aligned}
$$

where $x_{S C}=\left\{p_{i t}(m), u_{i t}, d_{j t}(m), u_{j t}, R_{i t}\right\}, x_{S C} \in U_{S C}$, and $U_{S C}=$ $\left\{x_{S C} \mid x_{S C}\right.$ satisfies $\left.(2)-(9)\right\}$, so the proposed UC model to minimize generalized social cost could be simplified as $\min _{x_{S C} \in U_{S C}} F_{S C}\left(x_{S C}\right)$.

Since the demand curves are deterministic and same for both models, $\forall x_{S C} \in U_{S C}, \exists x_{S W} \in U_{S W}$, where $x_{S W}=\left\{p_{i t}(m), u_{i t}, l_{j t}(m), v_{j t}, R_{i t}\right\}$, 
$l_{j t}(m)=d_{j t}^{\max }(m)-d_{j t}(m), v_{j t}=1-u_{j t}$, and $U_{S W}=\left\{x_{S W} \mid x_{S W}\right.$ satisfies $(2)-(9)$, substituted with $l_{j t}(m)$ and $\left.v_{j t}\right\}$. There is a one-to-one mapping relationship between $U_{S C}$ and $U_{S W}$.

Assume $\hat{x}_{S C}=\left\{\hat{p}_{i t}(m), \hat{u}_{i t}, \hat{d}_{j t}(m), \hat{u}_{j t}, \hat{R}_{i t}\right\}$ is the solution of the proposed UC model $\min _{x_{S C} \in U_{S C}} F_{S C}\left(x_{S C}\right)$, i.e., $F_{S C}\left(\hat{x}_{S C}\right) \leq F_{S C}\left(x_{S C}\right), \forall x_{S C} \in$ $U_{S C}$, substitute $\hat{d}_{j t}(m)$ and $\hat{u}_{j t}$ by $d_{j t}^{\max }(m)-\hat{l}_{j t}(m)$ and $1-\hat{v}_{j t}$, we obtain

$$
\begin{aligned}
F_{S C}\left(\hat{x}_{S C}\right)= & \sum_{t=1}^{N T} \sum_{i=1}^{N G}\left[\sum_{m=1}^{N I} \lambda_{i t}(m) \hat{p}_{i t}(m)+A_{i} \hat{u}_{i t}\right] \\
& +\sum_{t=1}^{N T} \sum_{j=1}^{N D}\left[\sum_{m=1}^{N J} m c_{j t}(m) d_{j t}^{\max }(m)+B_{j}\right] \\
& -\sum_{t=1}^{N T} \sum_{j=1}^{N D}\left[\sum_{m=1}^{N J} m c_{j t}(m) \hat{l}_{j t}(m)+B_{j} \hat{v}_{j t}\right] \\
& +\sum_{t=1}^{N T} \sum_{i=1}^{N G} S_{i t}\left(\hat{u}_{i t}, \hat{u}_{i, t-1}\right)+\sum_{t=1}^{N T} \sum_{i=1}^{N G} C_{i t} \hat{R}_{i t}
\end{aligned}
$$

Since $\sum_{t=1}^{N T} \sum_{j=1}^{N D}\left[\sum_{m=1}^{N J} m c_{j t}(m) d_{j t}^{\max }(m)+B_{j}\right]$ is total benefit of the whole demand curve, which can be expressed as a constant $C$, and the function of social welfare is

$$
\begin{aligned}
F_{S W}\left(x_{S W}\right)= & \sum_{t=1}^{N T} \sum_{j=1}^{N D}\left[\sum_{m=1}^{N J} m c_{j t}(m) l_{j t}(m)+B_{j} v_{j t}\right] \\
& -\sum_{t=1}^{N T} \sum_{i=1}^{N G}\left[\sum_{m=1}^{N I} \lambda_{i t}(m) p_{i t}(m)+A_{i} u_{i t}\right] \\
& -\sum_{t=1}^{N T} \sum_{i=1}^{N G} S_{i t}\left(u_{i t}, u_{i, t-1}\right)-\sum_{t=1}^{N T} \sum_{i=1}^{N G} C_{i t} R_{i t}
\end{aligned}
$$

We can obtain $F_{S C}\left(\hat{x}_{S C}\right)=C-F_{S W}\left(\hat{x}_{S W}\right)$, and $F_{S C}\left(x_{S C}\right)=C-F_{S W}\left(x_{S W}\right)$. Thus, $C-F_{S W}\left(\hat{x}_{S W}\right) \leq C-F_{S W}\left(x_{S W}\right), \forall x_{S W} \in U_{S W}$, i.e., $F_{S W}\left(\hat{x}_{S W}\right) \geq$ $F_{S W}\left(x_{S W}\right), \forall x_{S W} \in U_{S W}$. In other words, the solution of the proposed UC to minimize generalized social cost maximizes the social welfare of the tradition UC model at the same time. With deterministic demand curves, the proposed $\mathrm{UC}$ that minimizes generalized social cost is equivalent to the traditional UC that maximizes social welfare. 


\section{Appendix B. Proof of equivalence between worst case of minimiz- ing generalized social cost and best case of maximiz- ing social welfare with uncertain demand curves}

The generalized social cost of proposed robust UC under the worst scenario of demand price elasticity is

$$
\begin{aligned}
F_{S C}^{\prime}\left(x_{S C}\right)= & \sum_{t=1}^{N T} \sum_{i=1}^{N G}\left[\sum_{m=1}^{N I} \lambda_{i t}(m) p_{i t}(m)+A_{i} u_{i t}\right] \\
& +\sum_{t=1}^{N T} \sum_{j=1}^{N D}\left[\sum_{m=1}^{N J} m c_{j t}(m) d_{j t}(m)+B_{j} u_{j t}\right] \\
& +\sum_{t=1}^{N T} \sum_{i=1}^{N G}\left[S_{i t}\left(u_{i t}, u_{i, t-1}\right)+C_{i t} R_{i t}\right] \\
& +\sum_{(j t) \in J_{0}}\left\{\triangle \alpha_{j t}\left|u_{j t}\left(e_{j t}^{0}\right)^{2} / 2+\sum_{m=1}^{N J} d_{j t}(m)\left(e^{m-1}+e^{m}\right) / 2\right|\right\}
\end{aligned}
$$

where $x_{S C}=\left\{p_{i t}(m), u_{i t}, d_{j t}(m), u_{j t}, R_{i t}\right\}, x_{S C} \in U_{S C}$, and $U_{S C}=$ $\left\{x_{S C} \mid x_{S C}\right.$ satisfies (2) - (9) $\}$, so the proposed robust UC model to minimize generalized social cost considering all uncertain demand price elasticities could be simplified as $\min _{x_{S C} \in U_{S C}} F_{S C}^{\prime}\left(x_{S C}\right)$. Similarly, $\forall x_{S C} \in U_{S C}, \exists x_{S W} \in$ $U_{S W}$, where $x_{S W}=\left\{p_{i t}(m), u_{i t}, l_{j t}(m), v_{j t}, R_{i t}\right\}, l_{j t}(m)=d_{j t}^{\max }(m)-d_{j t}(m)$, $v_{j t}=1-u_{j t}$, and $U_{S W}=\left\{x_{S W} \mid x_{S W}\right.$ satisfies (2) - (9) substituted with $l_{j t}(m)$ and $\left.v_{j t}\right\}$. Assume $\hat{x}_{S C}=\left\{\hat{p}_{i t}(m), \hat{u}_{i t}, \hat{d}_{j t}(m), \hat{u}_{j t}, \hat{R}_{i t}\right\}$ is the solution of the proposed UC model $\min _{x_{S C} \in U_{S C}} F_{S C}^{\prime}\left(x_{S C}\right)$, i.e., $F_{S C}^{\prime}\left(\hat{x}_{S C}\right) \leq F_{S C}^{\prime}\left(x_{S C}\right), \forall x_{S C} \in$ $U_{S C}$, substitute $\hat{d}_{j t}(m)$ and $\hat{u}_{j t}$ by $d_{j t}^{\max }(m)-\hat{l}_{j t}(m)$ and $1-\hat{v}_{j t}$, we get 


$$
\begin{aligned}
F_{S C}^{\prime}\left(\hat{x}_{S C}\right)= & \sum_{t=1}^{N T} \sum_{i=1}^{N G}\left[\sum_{m=1}^{N I} \lambda_{i t}(m) \hat{p}_{i t}(m)+A_{i} \hat{u}_{i t}\right] \\
& +\sum_{t=1}^{N T} \sum_{j=1}^{N D}\left[\sum_{m=1}^{N J} m c_{j t}(m) d_{j t}^{\max }(m)+B_{j}\right] \\
& -\sum_{t=1}^{N T} \sum_{j=1}^{N D}\left[\sum_{m=1}^{N J} m c_{j t}(m) \hat{l}_{j t}(m)+B_{j} \hat{v}_{j t}\right] \\
& +\sum_{t=1}^{N T} \sum_{i=1}^{N G} S_{i t}\left(\hat{u}_{i t}, \hat{u}_{i, t-1}\right)+\sum_{t=1}^{N T} \sum_{i=1}^{N G} C_{i t} \hat{R}_{i t} \\
& +\sum_{(j t) \in J_{0}}\left\{\triangle \alpha_{j t}\left|\left(e_{j t}^{0}\right)^{2} / 2+\sum_{m=1}^{N J} d_{j t}^{m a x}(m)\left(e^{m-1}+e^{m}\right) / 2\right|\right\} \\
& -\sum_{(j t) \in J_{0}}\left\{\triangle \alpha_{j t}\left|\hat{v}_{j t}\left(e_{j t}^{0}\right)^{2} / 2+\sum_{m=1}^{N J} \hat{l}_{j t}(m)\left(e^{m-1}+e^{m}\right) / 2\right|\right\} \\
\text { Since } \sum_{(j t) \in J_{0}}\{ & \left.\triangle \alpha_{j t}\left|\left(e_{j t}^{0}\right)^{2} / 2+\sum_{m=1}^{N J} d_{j t}^{m a x}(m)\left(e^{m-1}+e^{m}\right) / 2\right|\right\}
\end{aligned}
$$
$\sum_{t=1}^{N T} \sum_{j=1}^{N D}\left[\sum_{m=1}^{N J} m c_{j t}(m) d_{j t}^{\max }(m)+B_{j}\right]$ are constants which can be expressed as $C_{1}$ and $C_{2}$, and the social welfare of robust UC to maximize social welfare under the best scenario of demand price elasiticity is

$$
\begin{aligned}
F_{S W}^{\prime}\left(x_{S W}\right)= & \sum_{t=1}^{N T} \sum_{j=1}^{N D}\left[\sum_{m=1}^{N J} m c_{j t}(m) l_{j t}(m)+B_{j} v_{j t}\right] \\
& -\sum_{t=1}^{N T} \sum_{i=1}^{N G}\left[\sum_{m=1}^{N I} \lambda_{i t}(m) p_{i t}(m)+A_{i} u_{i t}\right] \\
& -\sum_{t=1}^{N T} \sum_{i=1}^{N G} S_{i t}\left(u_{i t}, u_{i, t-1}\right)-\sum_{t=1}^{N T} \sum_{i=1}^{N G} C_{i t} R_{i t} \\
& +\sum_{(j t) \in J_{0}}\left\{\triangle \alpha_{j t}\left|v_{j t}\left(e_{j t}^{0}\right)^{2} / 2+\sum_{m=1}^{N J} l_{j t}(m)\left(e^{m-1}+e^{m}\right) / 2\right|\right\}
\end{aligned}
$$

We can obtain $F_{S C}^{\prime}\left(\hat{x}_{S C}\right)=C_{1}+C_{2}-F_{S W}^{\prime}\left(\hat{x}_{S W}\right)$, and $F_{S C}^{\prime}\left(x_{S C}\right)=C 1+$ 
$C 2-F_{S W}^{\prime}\left(x_{S W}\right)$. Thus, $C 1+C 2-F_{S W}^{\prime}\left(\hat{x}_{S W}\right) \leq C-F_{S W}^{\prime}\left(x_{S W}\right), \forall x_{S W} \in$ $U_{S W}$, i.e., $F_{S W}^{\prime}\left(\hat{x}_{S W}\right) \geq F_{S W}^{\prime}\left(x_{S W}\right), \forall x_{S W} \in U_{S W}$. In other words, the worst scenario in term of minimizing generalized social cost is the best scenario in terms of maximizing social welfare.

\section{Appendix C. Derivation of Robust UC Objective Function (15)}

Since only the last two lines of (14) are nonlinear, we only need to convert them into mixed linear form. First of all, we define variables:

$$
x_{j t}=u_{j t}\left(e_{j t}^{0}\right)^{2} / 2+\sum_{m=1}^{N_{J}} d_{j t}(m)\left(e^{m-1}+e^{m}\right) / 2 \quad \forall j t \in J_{0}
$$

Then, the last two lines of (14) can be expressed as:

$$
\begin{aligned}
\beta\left(x_{j t}\right) & =\max _{\left\{S_{0}\left|S_{0} \subseteq J_{0},\right| S_{0} \mid \leq \Gamma_{0}\right\}} \sum_{(j t) \in S_{0}} \triangle \alpha_{j t}\left|x_{j t}\right| \\
& =\max \left\{\sum_{j t \in J_{0}} \triangle \alpha_{j t}\left|x_{j t}\right| z_{0 j t}: \sum_{j t \in J_{0}} z_{0 j t} \leq \Gamma_{0}, 0 \leq z_{0 j t} \leq 1, \forall j t \in J_{0}\right\}
\end{aligned}
$$

By the property of strong duality, we can formulate the dual problem of (C.2) as (C.3), where $z_{0}$ is the dual variable of constraint $\sum_{j t \in J_{0}} z_{0 j t} \leq \Gamma_{0}, q_{j t}$ is the dual variable of constraint $z_{0 j t} \leq 1$ and $y_{j t}$ is auxiliary variable used to obtain equivalent linear expression.

$$
\begin{aligned}
\beta\left(x_{j t}\right)=\min & \left\{\sum_{j t \in J_{0}} q_{j t}+z_{0} \Gamma_{0}: z_{0}+q_{j t} \geq \triangle \alpha_{j t}\left|x_{j t}\right|, q_{j t} \geq 0, z_{0} \geq 0, \forall j t \in J_{0}\right\} \\
=\min & \left\{\sum_{j t \in J_{0}} q_{j t}+z_{0} \Gamma_{0}: z_{0}+q_{j t} \geq \triangle \alpha_{j t} y_{j t}, q_{j t} \geq 0, z_{0} \geq 0,\right. \\
& \left.-y_{j t} \leq x_{j t} \leq y_{j t}, y_{j t} \geq 0, \forall j t \in J_{0}\right\}
\end{aligned}
$$

Assume all $\alpha_{j t}$ are subject to uncertainty, substitute (C.3) into (14), we derived the objective function of robust UC in mixed integer linear form as (15).

\section{References}

[1] R. Baldick, Wind and energy markets: A case study of Texas, IEEE Systems J. 6(1) (2012) 27-34.

[2] S. N. Singh, J. Østergaard, Use of demand response in electricity markets: An overview and key issues, in: European Energy Market (EEM) Conf., June 2010, pp. 1-6. 
[3] F. Rahimi, A. Ipakchi, Demand response as a market resource under the smart grid paradigm, IEEE Trans. Smart Grid 1(1) (2010) 82-88.

[4] P. T. Baboli, M. P. Moghaddam, M. Eghbal, Present status and future trends in enabling demand response programs, in: IEEE PES Gen. Meet., Detroit, MI, USA, July 2011, pp. 1-6.

[5] J. Wang, N. E. Redondo, F. D. Galiana, Demand-side reserve offers in joint energy/reserve electricity markets, IEEE Trans. Power Syst. 18(4) (2003) 1300- 1306.

[6] D. S. Kirschen, G. Strbac, P. Cumperayot, D. Mendes, Factoring the elasticity of demand in electricity prices, IEEE Trans. Power Syst. 15(2) (2000) 612-617.

[7] J. Wang, S. Kennedy, J. Kirtley, A new wholesale bidding mechanism for enhanced demand response in smart grids, in: IEEE Innov. Smart Grid Technol. (ISGT) Conf., January 2010, pp. 1-8.

[8] C. Su, D. Kirschen, Quantifying the effect of demand response on electricity markets, IEEE Trans. Power Syst. 24(3) (2009) 1199-1207.

[9] K. Singh, N. P. Padhy, J. Sharma, Influence of price responsive demand shifting bidding on congestion and LMP in pool-based day-ahead electricity markets,IEEE Trans. Power Syst. 26(2) (2011) 886-896.

[10] A. Khodaei, M. Shahidehpour, S. Bahramirad, SCUC with hourly demand response considering intertemporal load characteristics, IEEE Trans. Smart Grid 2(3) (2011) 564-571.

[11] M. Parvania, M. Fotuhi-Firuzabad, Demand response scheduling by stochastic SCUC, IEEE Trans. Smart Grid 1(1) (2010) 89-98.

[12] E. Karangelos, F. Bouffard, Towards full integration of demand-side resources in joint forward energy/reserve electricity markets, IEEE Trans. Power Syst. 27(1) (2012) $280-289$.

[13] G. Liu, K. Tomsovic, A full demand response model in co-optimized energy and reserve market, Elect. Power Syst. Res. 111 (2014) 62-70.

[14] Federal Energy Regulatory Commission, Wholesale Competition in Regions With Organized Electric Markets, FERC Order No. 719 [Online]. Available: http://www.ferc.gov/whats-new/comm-meet/2008/101608/E-1.pdf.

[15] National Action Paln on Demand Response, Federal Energy Regulatory Commission, Jun. 2010. [Online]. Available: http://www.ferc.gov/legal/staff-reports/06-1710-demand-response.pdf

[16] R. Mukerji, Demand response in the NYISO markets, in: IEEE Power Systems Conf. and Expo. (PSCE), Phoenix, AZ, USA, March 2011, pp. 1-2. 
[17] R. Barth, H. Brand, P. Meibom, C. Weber, A stochastic unit-commitment model for the evaluation of the impacts of integration of large amounts of intermittent wind power, in: Probabilistic Methods Applied to Power Systems (PMAPS) Conf., June 2010, pp. 1-8.

[18] A. Tuohy, P. Meibom, E. Denny, M. O'Malley, Unit commitment for systems with significant wind penetration, IEEE Trans. Power Syst. 24(2) (2009) 592-601.

[19] P. Ruiz, C. Philbrick, E. Zak, K. Cheung, P. Sauer, Uncertainty management in the unit commitment problem, IEEE Trans. Power Syst. 24(2) (2013) 642-651.

[20] E. Constantinescu, V. Zavala, M. Rocklin, L. Sangmin, M. Anitescu, A computational framework for uncertainty quantification and stochastic optimization in unit commitment with wind power generation, IEEE Trans. Power Syst. 26(1) (2011) 431-441.

[21] F. Bouffard, F. Galiana, Stochastic security for operations planning with significant wind power generation, IEEE Trans. Power Syst. 23(2) (2008) 306-316.

[22] B. Ummels, M. Gibescu, E. Pelgrum, W. Kling, A. Brand, Impacts of wind power on thermal generation unit commitment and dispatch, IEEE Trans. Energy Convers., 22(1) (2007) 44-51.

[23] J. Wang, M. Shahidehpour, Z. Li, Security-constrained unit commitment with volatile wind power generation, IEEE Trans. Power Syst. 23(3) (2008) 1319-1327.

[24] Q. Wang, Y. Guan, J. Wang, A chance-constrained two-stage stochastic program for unit commitment with uncertain wind power output, IEEE Trans. Power Syst. 27(1) (2012) 206-215.

[25] R. Jiang, M. Zhang, G. Li, Y. Guan, Two-Stage Robust Power Grid Optimization Problem, Tech. Rep., 2011.

[26] D. Bertsimas, E. Litvinov, X. A. Sun, J. Zhao, T. Zheng, Adaptive robust optimization for the security constrained unit commitment problem, IEEE Trans. Power Syst. 28(1) (2013) 52-63.

[27] R. Jiang, J. Wang, Y. Guan, Robust unit commitment with wind power and pumped storage hydro, IEEE Trans. Power Syst. 27(2) (2012) 800-810.

[28] A. Street, F. Oliveira, J. Arroyo, Contingency-constrained unit commitment with n-K security criterion: A robust optimization approach, IEEE Trans. Power Syst. $26(3)$ (2011) 1581-1590.

[29] L. Baringo, A. J. Conejo, Offering strategy via robust optimization, IEEE Trans. Power Syst. 26(3) (2011) 1418-1425. 
[30] Q. Wang, J. Wang, Y. Guan, Stochastic unit commitment with uncertain demand response, IEEE Trans. Power Syst. 28(1) (2013) 562-563.

[31] C. Zhao, J. Wang, J. P. Watson, Y. Guan, Multi-stage robust unit commitment considering wind and demand response uncertainties, IEEE Trans. Power Syst. 28(3) (2013) 2708-2717.

[32] L. Zhao, B. Zeng, Robust unit commitment problem with demand response and wind energy, in: IEEE PES Gen. Meet., July 2012, pp. 1-8.

[33] O. Wolfgang, G. Doorman, Evaluating demand side measures in simulation models for the power market, Elect. Power Syst. Res. 81(3) (2011) 790-797.

[34] M. Ortega-Vazquez, Optimizing the spinning reserve requirements, Sch. Elect. Electron. Eng. Manchester, U.K.: Univ. Manchester, 2006, pp. 1-219 [Online]. Available: http://www.eee.manchester. ac.uk/research/groups/eeps/publications/reportstheses/aoe/ortegavazquez_PhD_2006.pdf.

[35] F. Aminifar, M. Fotuhi-Firuzabad, M. Shahidehpour, Unit commitment with probabilistic spinning reserve and interruptible load considerations, IEEE Trans. Power Syst. 24 (1) (2009) 388-397.

[36] W. G. Wood, Spinning reserve constrained static and dynamic economic dispatch, IEEE Trans. Power App. Syst. 101(2) (1982) 381-388.

[37] G. Liu, K. Tomsovic, Quantifying spinning reserve in systems with significant wind power penetration, IEEE Trans. Power Syst. 27(4) (2012) 2385-2392.

[38] M. Carrión, J.M. Arroyo, A computationally efficient mixed-integer linear formulation for the thermal unit commitment problem, IEEE Trans. Power Syst. 21 (3) (2006) 1371-1378.

[39] D. Bertsimas, M. Sim, Robust discrete optimization and network flows, Math. Program., Ser. B, 98 (2003) 49-71.

[40] C. Jonghe, B. F. Hobbs, R. Belmans, Optimal Generation Mix With Short-Term Demand Response and Wind Penetration, IEEE Trans. Power Syst. 27(2) (2012) 830-839.

[41] Reliability Test System Task Force, The IEEE Reliability Test System-1996. A report prepared by the Reliability Test System Task Force of the Application of Probability Methods Subcommittee. IEEE Trans. Power Syst. 14 (3) (1999) 1010-1020.

[42] C. Wang, M. Shahidehpour, Effects of ramp-rate limits on unit commitment and economic dispatch, IEEE Trans. Power Syst. 8 (3) (1993) 1341-1350.

[43] F. Bouffard, F. Galiana, An electricity market with a probabilistic spinning reserve criterion, IEEE Trans. Power Syst. 19(1)(2004) 300- 307. 